University of Wollongong

Research Online

Faculty of Engineering and Information

Faculty of Engineering and Information

Sciences - Papers: Part A

Sciences

$1-1-2012$

Replication of polypyrrole with photonic structures from butterfly wings as biosensor

Jie Tang

Shanghai Jiao Tong University

Shenmin Zhu

Shanghai Jiao Tong University

Zhixin Chen

University of Wollongong, zchen@uow.edu.au

Chuanliang Feng

State Key Laboratory of Metal Matrix Composites

Yanjun Shen

Shanghai Jiao Tong University

See next page for additional authors

Follow this and additional works at: https://ro.uow.edu.au/eispapers

Part of the Engineering Commons, and the Science and Technology Studies Commons

Research Online is the open access institutional repository for the University of Wollongong. For further information contact the UOW Library: research-pubs@uow.edu.au 


\title{
Replication of polypyrrole with photonic structures from butterfly wings as biosensor
}

\begin{abstract}
Polypyrrole (PPy) with photonic crystal structures were synthesized from Morpho butterfly wings using a two-step templating process. In the first step photonic crystal SiO2 butterfly wings were synthesized from Morpho butterfly wings and in the second step the SiO2 butterfly wings were used as templates for the replication of PPy butterfly wings using an in situ polymerization method. The SiO2 templates were then removed from the PPy butterfly wings using a HF solution. The hierarchical structures down to the nanometer level, especially the photonic crystal structures, were retained in the final PPy replicas, as evidenced directly by field-emission scanning electron microscope (FE-SEM) and transmission electron microscopy (TEM). The optical properties of the resultant PPy replicas were investigated using reflectance spectroscopy and the PPy replicas exhibit brilliant color due to Bragg diffraction through its ordered periodic structures. The preliminary biosensing application was investigated and it was found that the PPy replicas showed a much higher biological activity compared with PPy powders through their response to dopamine (DA), probably due to the hierarchical structures as well as controlled porosity inherited from Morpho butterfly wings. It is expected that our strategy will open up new avenues for the synthesis of functional polymers with photonic crystal structures, which may form applications as biosensors.
\end{abstract}

\section{Keywords}

biosensor, wings, butterfly, structures, polypyrrole, photonic, replication

\section{Disciplines}

Engineering | Science and Technology Studies

\section{Publication Details}

Tang, J., Zhu, S., Chen, Z., Feng, C., Shen, Y., Yao, F., Zhang, D., Moon, W. \& Song, D. (2012). Replication of polypyrrole with photonic structures from butterfly wings as biosensor. Materials Chemistry and Physics, 131 (3), 706-713.

\section{Authors}

Jie Tang, Shenmin Zhu, Zhixin Chen, Chuanliang Feng, Yanjun Shen, Fan Yao, Di Zhang, Won-Jin Moon, and Deok-Min Song 
Replication of polypyrrole with photonic structures from butterfly wings as biosensor Jie Tang ${ }^{a}$, Shenmin Zhu ${ }^{\mathrm{a}, *}$, Zhixin Chen ${ }^{\mathrm{b}}$, Chuanliang Feng ${ }^{\mathrm{a}}$, Yanjun Shen ${ }^{\mathrm{a}}$, Fan Yao ${ }^{\mathrm{a}}$, Di Zhang ${ }^{\mathrm{a}, *}$ and Won-Jin Moon ${ }^{\mathrm{c}}$

${ }^{\mathrm{a}}$ State Key Laboratory of Metal Matrix Composites, Shanghai Jiao Tong University, 800 Dongchuan Road, Shanghai 200240, P. R. China

${ }^{\mathrm{b}}$ Faculty of Engineering, University of Wollongong, Wollongong, NSW 2522, Australia

${ }^{c}$ Chonnam National University,300 YongBong-Dong, Buk-Gu, Gwang ju 500-757,Korea, Gwang ju Center, Korea Basic Science Institute

[*] Corresponding author.

E-mail: smzhu@sjtu.edu.cn, zhangdi@sjtu.edu.cn

Tel: +86-21-34202584; Fax: +86-21-34202749

\begin{abstract}
Photonic crystal polypyrrole (PPy) butterfly wings were synthesized from Morpho butterfly wings by using a two-step templating process. In the first step photonic crystal $\mathrm{SiO}_{2}$ butterfly wings were synthesized from Morpho butterfly wings and in the second step the obtained $\mathrm{SiO}_{2}$ replicas were used as templates for the duplication of PPy butterfly wings by using an in situ polymerization method. The $\mathrm{SiO}_{2}$ templates were then removed from the PPy butterfly wings using a HF solution. The precise structures of the replicas were characterized by using field emission scanning electron microscopy (FE-SEM), transmission electron microscopy (TEM). Molecular structure and the composition of the PPy replicas were further characterized by Fourier infrared spectroscopy (FT-IR) and energy-dispersive X-ray spectroscopy (EDS), respectively. It was confirmed that the photonic crystal PPy replicas were made of pure PPy. The optical properties of
\end{abstract}


the resultant PPy replicas were investigated by using reflectance spectroscopy and the PPy replicas exhibit brilliant color due to Bragg diffraction through its ordered periodic structures. The primary response of the replicas to dopamine (DA) as a biosensor was investigated and it was found that the PPy replicas showed a much higher biological activity compared with PPy powders, probably due to the hierarchical structures as well as controlled porosity inherited from Morpho butterfly wings. It is expected that our strategy will open up new avenues for the synthesis of functional polymers with photonic crystal structures, which can form the basis of promising applications as biosensors.

Keywords: Polymers, Ultrasonic techniques, Nanostructures, Electrochemical properties

\section{Introduction}

Photonic crystals (PC) have been extensively studied because of their potential applications in optical communication, smart windows, memory devices, and biosensors. Templatings from the sedimentation of monodispersed silica or polystyrene spheres, have been used to fabricate photonic crystals with opal or inverse-opal structure, including semiconductors [1, 2], metals [3], polymers [4, 5] and hydrogels [6]. However, the synthesis of the ordered periodic dielectric structures relies on the assembling of colloidal crystals through the vertical deposition-evaporation method, which is not easy to control. In fact, nature provides abundant variety of structures at micro and nanometric scale with functional properties and is sources of great inspiration for numerous functional materials. For example, a specific structure of lotus leaves can give them super-hydrophobicity or self-cleaning properties [7]. Photonic crystal structures of Morpho butterfly wings have led to diversity of colors and patterns [8]. The biological organizations produced by self-assembly of 
highly ordered functional units provide us numerous opportunities in producing a wide range of photonic structural materials. Several nanostructural fabrication techniques have been developed to replicate natural photonic structures. They include electron beam lithography, focused ion-beam chemical vapor deposition [9], chemical vapor deposition [10], atomic layer deposition [11], the conformal-evaporated-film-by-rotation technique and soaking method [12]. In 2008, we successfully developed a new technology of using ultrasonic waves to replicate natural hierarchical structures in manganese oxide [13]. We extended this general facile technique to the synthesis of photonic structures in $\mathrm{TiO}_{2}, \mathrm{SnO}_{2}$ and $\mathrm{SiO}_{2}$ [14].

However, attempts to prepare polymer photonic or hierarchical replicas using these approaches had often failed. Generally the formation of pores and tiny structural details in polymers is not probable thermodynamically. Even when they are formed, the pores tend to be closed or fractured by plastic deformation of the walls. Another general hindrance for the nanostructuration of polymers is depletion, i.e. the entropic restriction of conformations in the nanoconfinement of particles and thin walls. Therefore, to obtain an organic material with interconnected meso- or microporous structures normally requires a stiff or highly cross-linked polymer system [15-17]. Caruso et al. have successfully developed polypyrrole (PPy) inverse opals for biosensing $[4,18]$ and fluorescence control [19]. However, the exact replication of PPy with hierarchical and photonic crystal structures from biological species is still a tough task, since biological structures are tremendously complex and consist of 3D interconnected macro, meso and nanoporous. In 2008, Saison et al reported the replication of butterfly wings with poly(dimethylsiloxane) (PDMS), unfortunately, only the surface structures was replicated by this casting process [20]. The general restrictions of this approach still hold and mutual compatibility of the template with both the 
monomer and the polymer phase is one of the many problems need to be addressed.

Inspired by our previous work on the precise replication of $\mathrm{SiO}_{2}$ photonic structures from butterfly wings, herein, we present a two-step template-directed approach for the fabrication of PPy with predetermined photonic and hierarchical structures of Morpho butterfly wings. As a conducting polymer, PPy has been widely explored for a comprehensive range of applications in biosensors [21, 22], gas sensors [23], microactuators [24], electrochemical supercapacitor [25] for its good environmental stability, facile synthesis, and higher conductivity. In particular, PPy has gained a distinguished popularity in biosensor applications because of its good biocompatibility, high sensitivity and fast response [26]. It is conceivable that electrical activity of PPy combined with obvious advantages of photonic crystals $[27,28]$ in sensitive biomolecular detection, will greatly improve its biosensor characteristics.

\section{Experimental}

\subsection{Synthesis of biomorphic PPy}

The Morpho butterfly wings we chose as biotemplates were supplied by Shanghai Natural Wild-Insect Kingdom Co., Ltd. Analytic grade reagents $\mathrm{HCl}, \mathrm{NaOH}, \mathrm{FeCl}_{3}, \mathrm{HF}$, alcohol, tetraethyl orthosilicate (TEOS), pyrrole were purchased from Shanghai Chemical Company. Pyrrole was distilled under reduced pressure and stored at low temperatures. The detailed processing is described as below: firstly, the wings pretreated with $6 \% \mathrm{HCl}$ and $10 \% \mathrm{NaOH}$ were carefully dipped into the ethanol/water/TEOS/HCl solution with the molar ratio of 3:12:1:0.03 and ultra-sonicated at room temperature for a period of $3 \mathrm{~h}$ using a high-intensity ultrasonic probe (Ti horn, $20 \mathrm{kHz}, 200$ $\mathrm{W} / \mathrm{cm}^{2}$ ). After the ultrasonic processing, the butterfly wings were taken out and washed three times 
with alcohol and dried in air. Calcination was performed at $450^{\circ} \mathrm{C}$ for $3 \mathrm{~h}$, then the chitin substrates were removed by reaction with air, leaving $\mathrm{SiO}_{2}$ in the form of ceramic butterfly wings. Secondly, the $\mathrm{SiO}_{2}$ replicas were placed on a microslide and dipped into $1-2 \mathrm{~mL}$ of pyrrole, and then in $0.1 \mathrm{~mL}$ of $0.21 \mathrm{M} \mathrm{FeCl}_{3}$. Capillary forces drew the liquid monomer into the void spaces between the silica particles. Further polymerization was carried out at $65^{\circ} \mathrm{C}$ for $3 \mathrm{~h}$. Finally, the microslide was soaked in a $4 \% \mathrm{HF}$ solution for $24 \mathrm{~h}$ to remove the $\mathrm{SiO}_{2}$. The resultant replicas thus prepared are denoted as PPy replica. For comparison, PPy powders without biological structures were synthesized in the same way, but without using $\mathrm{SiO}_{2}$ replicas as templates.

\subsection{Characterization}

The morphologies and microstructures of the resultant replicas were characterized using a JEOL JSM-6360LV field-emission scanning electron microscope (FE-SEM) equipped with a JED2300 energy-dispersive X-ray spectrometer (EDS) system. Transmission electron microscopy (TEM) was conducted on a JEOL 2011F. TEM specimens were prepared by grinding the synthesized samples into power with a mortar and pestle, after which the powder was dispersed in pure ethanol and picked up by holey carbon films on copper grids. Fourier transform-infrared (FT-IR) measurements were recorded with a PE Paragon 1000 spectrophotometer and the spectra were obtained from $\mathrm{KBr}$ pellets. The micrographs of the replicas were taken using a digital optical microscope VHX-600, Keyence.

\subsection{Measurement of biomolecule sensing properties}

Cyclic voltammetry (CV) was performed on a CHI 660D computer electrochemical workstation

(Chen Hua Instruments Co., Shanghai, China) with a conventional three-electrode system, which was composed of a working electrode, a platinum slice counter electrode and a saturated calomel 
reference electrode (SCE), respectively. A glassy carbon electrode (GCE, $\Phi 3.0 \mathrm{~mm}$ ) served as working electrode. Dopamine (DA) and nafion (Sigma-Aldrich) were used as received without further purification. The phosphate buffer solution (PBS, $0.10 \mathrm{M}, \mathrm{PH}=7$ ) was prepared by mixing $\mathrm{NaH}_{2} \mathrm{PO}_{4}$ and $\mathrm{Na}_{2} \mathrm{HPO}_{4}$. DA solution used in all experiments was freshly prepared. All solutions were prepared with doubly distilled water. Nano gold particles were introduced to the surface of the PPy replicas and the prepared PPy powder to improve the conductivity of the two materials. The detailed process was described as below: the PPy replicas as well as PPy powders were grinded and dispersed in water evenly, $10 \mathrm{~mL}$ of $0.01 \%$ gold (III) chloride trihydrate $\left(\mathrm{HAuCl}_{4} \cdot 3 \mathrm{H}_{2} \mathrm{O}\right.$, Shanghai Chemical Company) was added into the PPy suspension and heated to boiling, $1.25 \mathrm{~mL}$ of $1 \%$ sodium citrate dihydrate $\left(\mathrm{C}_{6} \mathrm{H}_{5} \mathrm{Na}_{3} \mathrm{O}_{7} \cdot 2 \mathrm{H}_{2} \mathrm{O}\right.$, Shanghai Chemical Company) was trickled into the boiling solution and the mixed solution was boiled for $0.5 \mathrm{~h}$. The above solution was then dropped on the surface of GCE and then $5 \mu \mathrm{L}$ of $1 \%$ nafion/alcohol solution after it was dried. The PPy modified GCE electrodes were investigated using CV in PBS containing $5.0 \times 10^{-3} \mathrm{M}$ DA.

\section{Results and Discussion}

The replication process is described in Scheme 1: Firstly, high-quality $\mathrm{SiO}_{2} \mathrm{PC}$ replicas made from Morpho butterfly wings was prepared by a sonochemical method as described before [14]. Then, the resultant $\mathrm{SiO}_{2}$ PC replica was dipped into a certain amount of pyrrole monomer. Subsequent adding $\mathrm{FeCl}_{3}$, resulted in the polymerization of pyrrole. After soaking in HF solution, the $\mathrm{SiO}_{2}$ template was removed. The replicas thus prepared are assigned as PPy replica.

(Scheme 1)

Secondary electron images of the original butterfly wing are shown in Fig. 1a-b. Fig. 1a reveals 
that the overlapping scales possess an overall rectangular shape with pointed tips. The scale surface is composed of thirty five to forty rows of lamellae with an almost identical interspacing. A closer view in Fig. $1 \mathrm{~b}$ indicates that the porous architecture of one such scale is comprised of parallel ridges, spaced several microns apart and aligned along the scale length. The lamellae are connected by cross-ribs and each cross rib is supported by one or two legs standing on the bottom surface of the scale so that there is a $1 \mu \mathrm{m}$ gap between the lamellae and the bottom surface of the scales.

Shown in Fig. 1c, the $\mathrm{SiO}_{2}$ replicas have the same shape, orientation and distribution as their 'parent' scales. The tile-like arrangement of the scales and the ridges with nanoscale ribs were retained in the $\mathrm{SiO}_{2}$ replicas after being calcined at $450^{\circ} \mathrm{C}$ for $3 \mathrm{~h}$. An extremely good replication of the fine detail of the original scale structures can clearly be seen. The longitudinal ridges are approximately $2 \mu \mathrm{m}$ apart, almost the same as the original butterfly wing demonstrating no obvious shrinkage during the calcination. The transverse ribs between the ridges have spaces between them in the region $50 \mathrm{~nm}$. TEM images (Fig. 1e-f) also reveal that the main and sub-ribs on the lamellae were well preserved without any obvious distortion. This structure is an inverse two dimensional (2D) photonic crystal slab, where periodic air spaces with particular shapes were confined and surrounded by a dielectric outer shell.

\section{(Figure 1)}

The morphology of the PPy replicas was investigated by SEM and the results are shown in Fig. 2a-d. It is clear that a good detail of the original scale structures were retained in the PPy replicas. Fig. 2a-b show that the PPy replica maintained the overall scale shape, parallel ridges and nanoscale ribs of the $\mathrm{SiO}_{2}$ template. Higher magnification SEM images (Fig. 2c-d) reveal that the inter-lamellae spacing are approximately $1.5 \mu \mathrm{m}$ for the PPy replica, which indicates that the overall 
dimension is reduced by $25 \%$ due to the shrinkage of PPy molecular chains during the polymerization process. Each of the cross-ribs has one or two legs standing on the bottom surface and exhibiting a hollow body. Between the ridges, the nanoscale ribs also exhibit a periodic lattice that extends from the surface toward the scale substrate beneath.

\section{(Figure 2)}

Fig. 2e-f show the morphology of the PPy replicas in TEM. Fig. 2e shows the microstructure of the PPy replica at a low magnification. The dark and dense walls are the ridges orderly ranged like parallel tubes, consistent with the results of SEM. As the technique was implemented at room temperature, the spatial features of the original butterfly wing were preserved without any obvious distortion during the replication (Fig. 2e). High magnification image in Fig. $2 \mathrm{f}$ further reveals that the well-organized parallel ridges were composed of numerous small particles down to nanometers. EDS microanalyses of the $\mathrm{SiO}_{2}$ and PPy replicas confirm that the PPy replica is mainly composed of carbon and nitrogen, corresponding to the chemical composition of PPy, with a small amount of fluorine, silicon and gold, which means that almost the entire $\mathrm{SiO}_{2}$ template was removed by the soaking in the HF solution (Fig. 2g). Fluorine was introduced when the samples were soaked in the HF solution and gold was due to the gold coating to minimize charging effects. It is reasonable to conclude that the unique morphological features of the butterfly wings, especially down to the nanometer level, were maintained in the final PPy replicas.

The chemical composition and the molecular structure of the PPy replicas, together with the $\mathrm{SiO}_{2}$ template and the PPy powder fabricated without using the $\mathrm{SiO}_{2}$ templates, were further investigated by using FTIR analysis (Fig. 3). The $\mathrm{SiO}_{2}$ template shows the characteristic absorption bands of $\mathrm{Si}-\mathrm{O}-\mathrm{Si}$ bond at $1090 \mathrm{~cm}^{-1}$. The absence of this absorption band in the FTIR spectrum of the PPy 
replica further confirms that the $\mathrm{SiO}_{2}$ templates were removed by the $\mathrm{HF}$ solution soaking. No obvious difference was observed in the FTIR spectra of the PPy replicas and PPy powders. The peaks centered at 1175,1457 and $1549 \mathrm{~cm}^{-1}$ were assigned to $\mathrm{C}-\mathrm{N}, \mathrm{C}-\mathrm{C}$ and $\mathrm{C}=\mathrm{C}$ backbone stretching vibrations, respectively and the peaks at 1303 and $1040 \mathrm{~cm}^{-1}$ were originated from C-H in-plane vibration. These peaks of the two PPy samples are all consistent with the characteristic of pure PPy $[29,30]$.

\section{(Figure 3)}

In contrast to nanostructured inorganic materials, the introduction of a nanostructure into organic materials is restricted because of the more stringent requirements on the resulting framework. In general, polymer chains pack space efficiently, because polymer macromolecules can bend and twist to maximize intermolecular interactions. Therefore, the maintenance of, for example, an interconnected meso- or microporosity in an organic material normally requires a stiff and highly crosslinked system. For comparison, we used poly N-isopropyl acrylamide (PNIPA), a relatively flexible polymer, to replicate the structures of Morpho butterfly wings in a similar process as that of the replication of the PPy. The SEM images indicate that the overall structure of a butterfly wing was obtained in the PNIPA replica (see Fig. S1 $\dagger$ ). However, the hierarchical structures, especially the photonic crystal structures, were not kept during the replication and almost all the cross-ribs were torn apart due to the motion and shrink of the molecular chains and plastic deformation of the walls after the remove of the $\mathrm{SiO}_{2}$ template. That means the final structures are interesting but far from the starting structure. This proved that a direct templating and in-situ polymerization is usually not suitable for the soft polymers.

Fig. 4 shows colored optical micrographs of the original butterfly wings, $\mathrm{SiO}_{2}$ templates and PPy 
replicas. Reflection measurements were performed between near ultraviolet and near infrared wavelength regions to reveal the interactions of the replicated structures with light. In the UV region, the original butterfly wing has a strongest reflection peak at $312 \mathrm{~nm}$. The relatively strong reflectance in the short visible wavelength region agrees with its natural violet color observed under an optical microscope (Fig. 4a). The $\mathrm{SiO}_{2}$ replica shows a broad and red shifted reflection peak at around 500-700 nm which is consistent with the orange/pink color observed under an optical microscope (Fig. 4b). The reflectance spectrum from the PPy replica (Fig. 4e shows that it has two strong reflection peaks at around 490-580 and 600-650 nm repctively, which also are consistent with the green/yellow color obtained under an optical microscope (Fig. 4c-d). These reflections or colors are due to the periodicity of the photonic crystal inherited from the $\mathrm{SiO}_{2}$ template or butterfly wings. All the colors of the Morpho butterfly wings and the replicas are mainly physical colors and attribute to optical interference involving photonic crystal structures, hierarchical structures (lamellae) and ordered mesopores structures (reticular network). Therefore, this study offers a simple and effective route for synthesizing biomorphic stiff polymers with photonic crystal structures.

\section{(Figure 4)}

Sensing properties. Applications of materials with photonic crystal structures to biosensors have attracted a great deal of interest. The inverse opal film that contains platinum nanoparticles showed improved electrocatalytic activity toward glucose oxidation [31]. Polyaniline (PANI) doped with poly(styrene sulfonate) (PSS) inverse opals for use as biosensor has been reported [32]. It is reasonable to expect the PPy replicas thus prepared with the promise of high surface area as well as controlled porosity due to their photonic crystal structure, would be ideal candidates for biosensors. 
The response of the biosensor to DA was used to characterize the biological sensibility of the PPy replica. Fig. 5 shows the cyclic voltammograms $(\mathrm{CVs})$ of $5.0 \times 10^{-3} \mathrm{M} \mathrm{DA}$ in $\mathrm{pH} 7.0$ phosphate buffer solution (PBS) with a scanning rate of $50 \mathrm{mV} / \mathrm{s}$, corresponding to a bare glassy carbon electrode (GCE) (curve a), PPy replica-Au/GCE (curve b) and common PPy-Au/GCE (curve c), respectively. The bare glassy carbon electrode showed a potential gap about $\Delta \mathrm{E}_{\mathrm{p}}=0.275 \mathrm{~V}$ between the redox peaks, with a peak redox current of $13 \mu \mathrm{A}$, illustrating that DA could exchange electrons with the electrode. After incubating the electrode with powder (PPy-Au/GCE), the peak redox current decreased to $4 \mu \mathrm{A}$, and potentials shifted to -0.05 and $0.45 \mathrm{~V}$. An obvious increase of the peak to peak separation was observed, suggesting the presence of PPy powder retarded the interfacial electron-transfer kinetics of DA at the electrode. Interestingly, the potential gap of PPy replica-Au/GCE drops to $\Delta \mathrm{E}_{\mathrm{p}}=0.222 \mathrm{~V}$, with cathodic potential at $0.032 \mathrm{~V}$ and anodic potential at $0.254 \mathrm{~V}$. This indicates that in PPy replica-Au/GCE, the direct electron transfer between DA and electrode is greatly enhanced as compared with other two electrodes: GCE and PPy-Au/GCE. The hierarchical structures of the PPy replica must provide a favorable microenvironment for DA to exchange electrons with the electrode and facilitate a high rate of electron transportation. It has been demonstrated that the biosensor characteristics are related to the effective surface area of the working electrode [33]. Inverse opal PANI composite has been reported to have a much higher electrocatalytic efficiency for the oxidation of reduced b-nicotinamide adenine dinucleotide $(\mathrm{NADH})$, than that of an unpatterned film [34]. Because the response of a biosensor depends on the interaction of an analyte with an immobilized capture ligand on the sensor surface. The increase of the surface area of a sensor will improve the sensitivity through a higher density of the capture ligand. The PPy replica has a large effective surface areabecause of its hierarchical structures and 
ordered porosity, which provide great accessibility for the biomolecules thus enhance the accumulation ability and catalytic efficiency [35]. Furthermore, the nano gold particles would disperse not only on the surface but also inside the pores of the PPy replicas due to the interconnected hierarchical structures. Thus, there is no surprise to see that the average peak current at PPy replica-Au/GCE electrode was about 3- and 9-fold higher than that at the bare GCE (curve a) and PPy-Au/GCE (curve c), respectively. The PPy butterfly wings replicas are clearly a very promising candidate for effectively biosensors.

\section{(Figure 5)}

\section{Conclusions}

The replication of PPy with photonic crystal structures from Morpho butterfly wings was achieved by using a two-step templating and in-situ polymerization approach. In this process $\mathrm{SiO}_{2}$ replica fabricated from butterfly wings was used as a template. The in-situ polymerization was achieved through the impregnation of the interstitial channels of the template with pyrrole monomer and polymerization. The subsequent removal of the $\mathrm{SiO}_{2}$ template with a $\mathrm{HF}$ solution resulted in the PPy PC, which replicated not only the outer but also the inner and more complex surfaces of the butterfly wings. The primary response of the replicas to dopamine (DA) as a biosensor was investigated and it was found that the PPy replicas showed nearly 10 times better sensitivity than that of the PPy powder, attributing to the controlled porosity and hierarchical structures inherited from Morpho butterfly wings. We anticipate that our strategy will open up new avenues for synthesis of electrically conductive polymers with photonic structures which can hardly be produced by using an artificial assembly synthesis route. 
Acknowledgments: The authors gratefully acknowledge the financial support of this research by the National Science Foundation of China (Nos. 50573013, 50772067), Shanghai Science and Technology Committee (Nos.06PJ14063, 07DJ14001), Program for New Century Excellent Talents in University, and Sino-French Project of MOST of China (No.2009DFA52410). We also thank SJTU Instrument Analysis Center for the measurements.

\section{References}

[1] Y.A. Vlasov, X.Z. Bo, J.C. Sturm, D.J. Norris, Nature, 414 (2001) 289.

[2] A. Blanco, E. Chomski, S. Grabtchak, M. Ibisate, S. John, S.W. Leonard, C. Lopez, F. Meseguer, H. Miguez, J.P. Mondia, G.A. Ozin, O. Toader, H.M. van Driel, Nature, 405 (2000) 437

[3] P. Jiang, J. Cizeron, J.F. Bertone, V.L. Colvin, J Am Chem Soc, 121 (1999) 7957.

[4] T. Cassagneau, F. Caruso, Adv Mater, 14 (2002) 34.

[5] H. Fudouzi, Y.N. Xia, Adv Mater, 15 (2003) 892.

[6] J.H. Holtz, S.A. Asher, Nature, 389 (1997) 829.

[7] M.H. Sun, C.X. Luo, L.P. Xu, H. Ji, O.Y. Qi, D.P. Yu, Y. Chen, Langmuir, 21 (2005) 8978.

[8] J.Y. Huang, X.D. Wang, Z.L. Wang, Nano Lett, 6 (2006) 2325.

[9] K. Watanabe, T. Hoshino, K. Kanda, Y. Haruyama, T. Kaito, S. Matsui, J Vac Sci Technol B, 23 (2005) 570.

[10] J.Z. Zhang, Z.Z. Gu, H.H. Chen, A. Fujishima, O. Sato, J Nanosci Nanotechno, 6 (2006) 1173.

[11] D.P. Gaillot, O. Deparis, V. Welch, B.K. Wagner, J.P. Vigneron, C.J. Summers, Phys Rev E, 78 (2008) 031922.

[12] J.G. Huang, T. Kunitake, J Am Chem Soc, 125 (2003) 11834.

[13] S.M. Zhu, D. Zhang, Z.Q. Li, H. Furukawa, Z.X. Chen, Langmuir, 24 (2008) 6292.

[14] S.M. Zhu, D. Zhang, Z.X. Chen, J.J. Gu, W.F. Li, H.B. Jiang, G. Zhou, Nanotechnology, 20 (2009) 315303.

[15] P. Jiang, K.S. Hwang, D.M. Mittleman, J.F. Bertone, V.L. Colvin, J Am Chem Soc, 121 (1999) 11630.

[16] S.A. Johnson, P.J. Ollivier, T.E. Mallouk, Science, 283 (1999) 963.

[17] J.Y. Wang, Y. Cao, Y. Feng, F. Yin, J.P. Gao, Adv Mater, 19 (2007) 3865.

[18] T. Cassagneau, F. Caruso, Adv Mater, 14 (2002) 1837.

[19] A.M. Yu, F. Meiser, T. Cassagneau, F. Caruso, Nano Lett, 4 (2004) 177.

[20] T. Saison, C. Peroz, V. Chauveau, S. Berthier, E. Sondergard, H. Arribart, Bioinspir Biomim, 3 (2008) 046004.

[21] J.C. Vidal, E. Garcia, J.R. Castillo, Anal Chim Acta, 385 (1999) 213.

[22] T.E. Campbell, A.J. Hodgson, G.G. Wallace, Electroanal, 11 (1999) 215.

[23] H.P. de Oliveira, A.H.P. de Oliveira, G.F. Tavares, J.J.F. Albuquerque, C.A.S. Andrade, Polimeros, 20 (2010) 253. 
[24] E. Smela, J Micromech Microeng, 9 (1999) 1.

[25] C. Yang, P. Liu, Synthetic Met, 160 (2010) 768.

[26] B.D. Malhotra, A. Chaubey, S.P. Singh, Anal Chim Acta, 578 (2006) 59.

[27] B.T. Cunningham, L. Laing, Expert Rev Proteomic, 3 (2006) 271.

[28] X.D. Fan, I.M. White, S.I. Shopoua, H.Y. Zhu, J.D. Suter, Y.Z. Sun, Anal Chim Acta, 620 (2008) 8.

[29] C. He, C.H. Yang, Y.F. Li, Synthetic Met, 139 (2003) 539.

[30] Y. Liu, Y. Chu, L.K. Yang, Mater Chem Phys, 98 (2006) 304.

[31] Y.Y. Song, D. Zhang, W. Gao, X.H. Xia, Chem-Eur J, 11 (2005) 2177.

[32] X.L. Yang, Y. Jin, Y.H. Zhu, L.H. Tang, C.Z. Li, J Electrochem Soc, 155 (2008) J23.

[33] E.M.I.M. Ekanayake, D.M.G. Preethichandra, K. Kaneto, Sensor Actuat B-Chem, 132 (2008) 166.

[34] S.J. Tian, J.J. Wang, U. Jonas, W. Knoll, Chem Mater, 17 (2005) 5726.

[35] H.M. Cao, Y.H. Zhu, L.H. Tang, X.L. Yang, C.Z. Li, Electroanal, 20 (2008) 2223.

\section{Captions of Figures:}

Scheme 1 The synthesis process of replication of butterfly wing in PPy.

Figure 1 (a) and (b) SEM images of butterfly wing scales, (c) and (d) SEM images of $\mathrm{SiO}_{2}$ replica, (e) and (f) TEM images of $\mathrm{SiO}_{2}$ replica and (g) an EDS spectrum obtained from $\mathrm{SiO}_{2}$ replica.

Figure 2 (a)-(d) SEM images of PPy replica, (e) and (f) TEM images of PPy replica and (g) an EDS 
spectrum obtained from PPy replica.

Figure 3 FT-IR spectra of $\mathrm{SiO}_{2}$ replica and PPy with different microstructures. (a) $\mathrm{SiO}_{2}$ replica, (b) PPy replica with hierarchical and photonic crystal structures and (c) PPy particles synthesized in a common way.

Figure 4 (a)-(d) Optical images of original butterfly wing, $\mathrm{SiO}_{2}$ replica and PPy replica, (c) and (d) optical images of PPy replica with different magnification and (e) reflectance results of original butterfly wings, $\mathrm{SiO}_{2}$ replica and PPy replica.

Figure 5 Cyclic voltammograms of 1.0x10 ${ }^{-3} \mathrm{M}$ DA at (a) bare GCE, (b) PPy replica-Au /GCE and (c) common PPy-Au /GCE. 


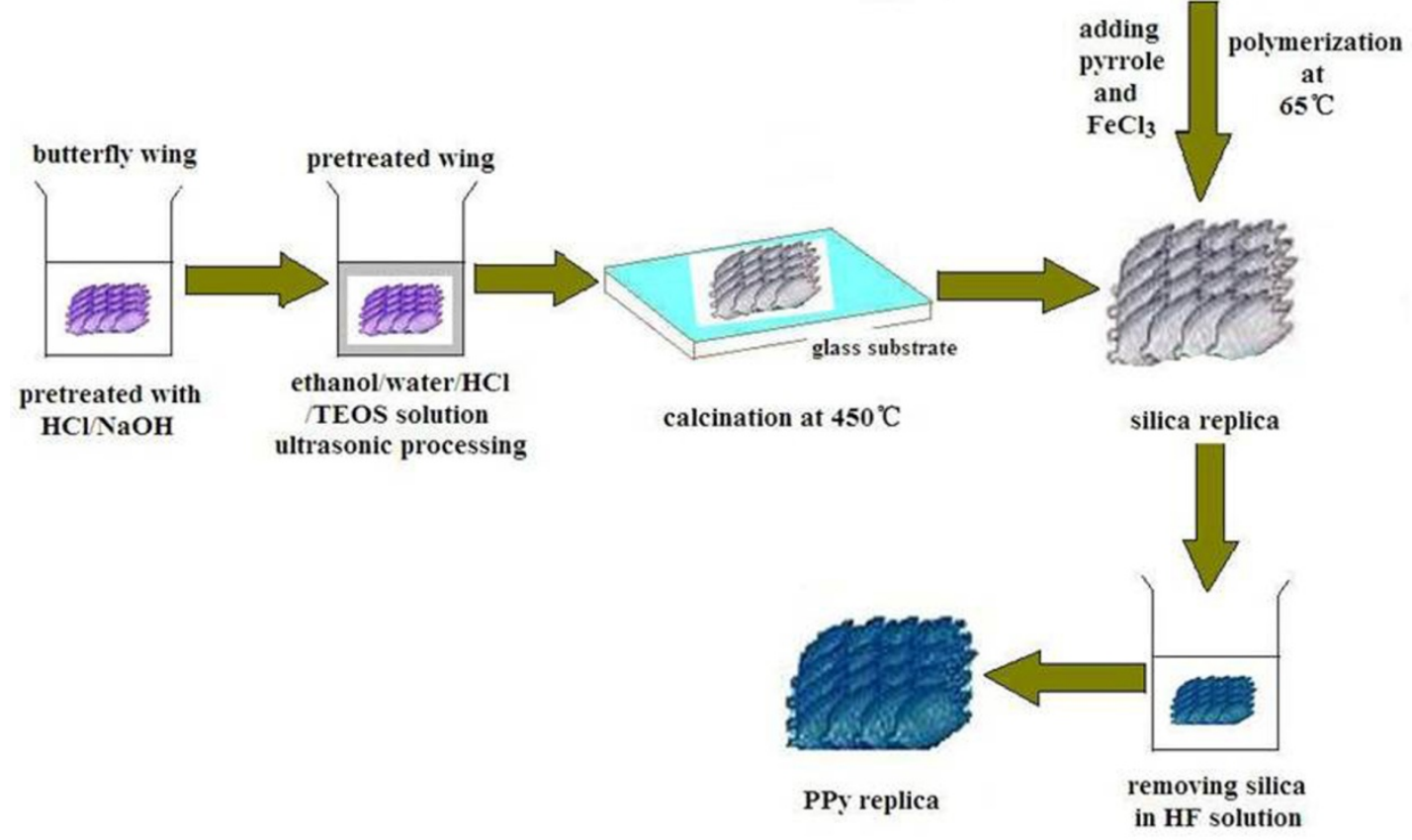

Scheme 1 

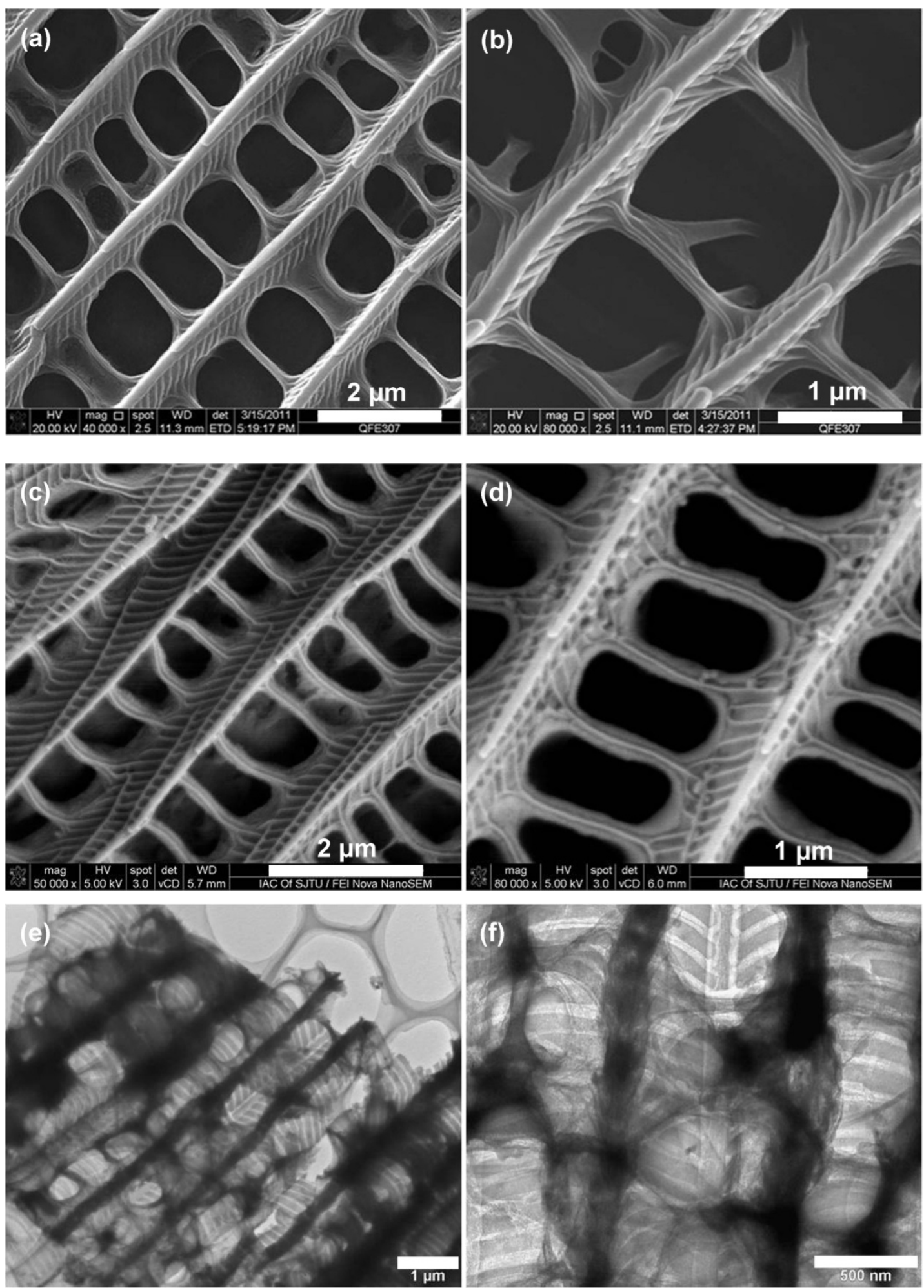


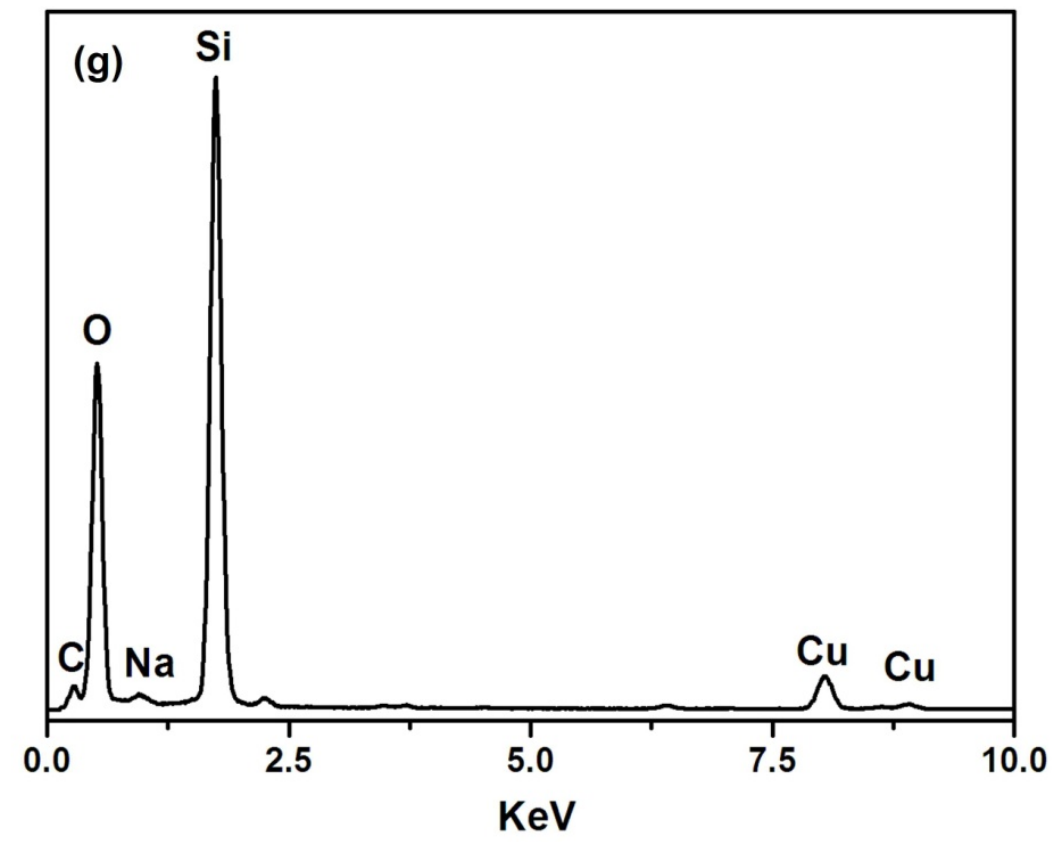

Figure 1 

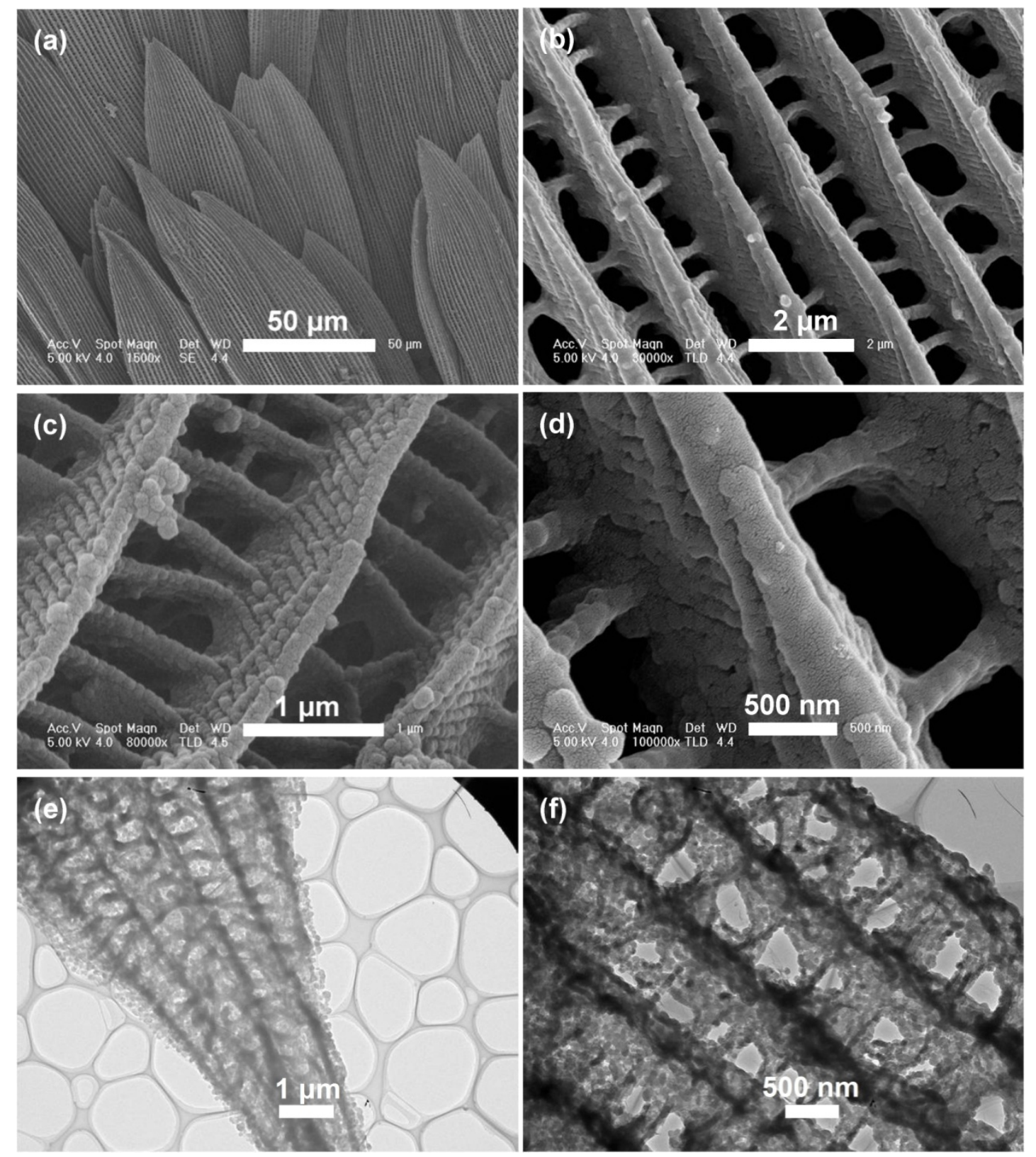


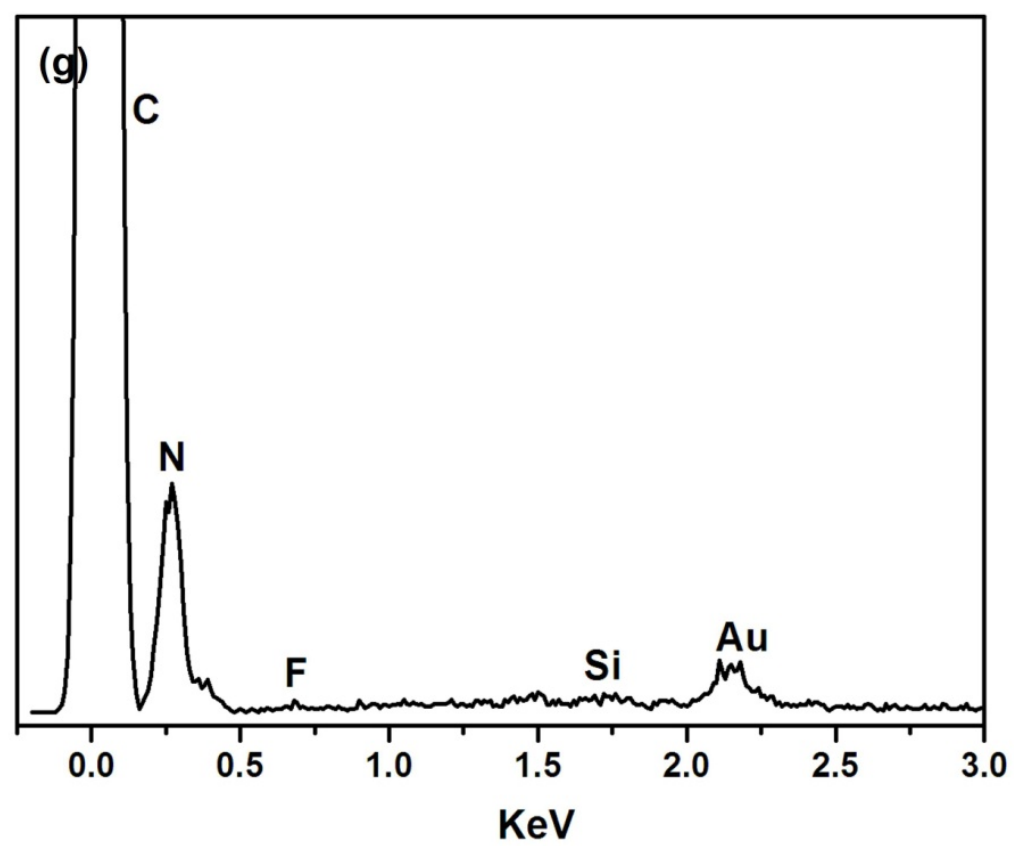

Figure 2 


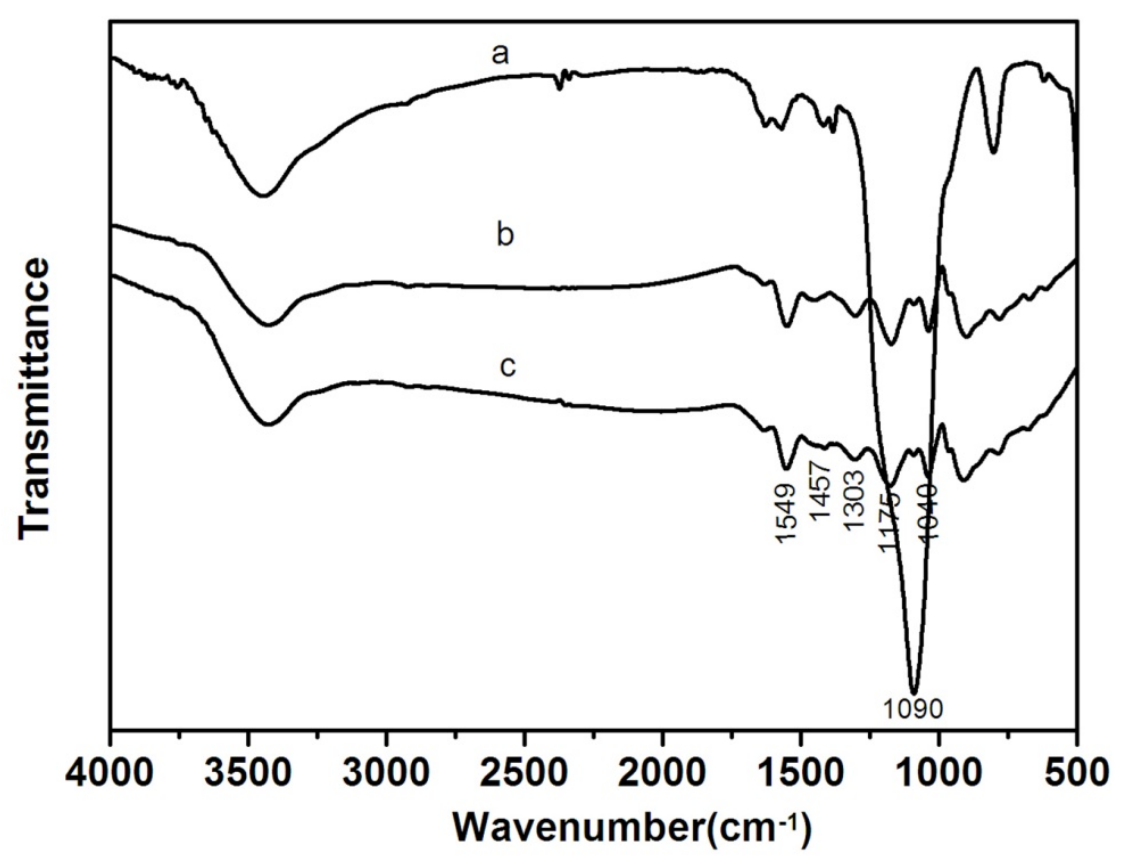

Figure 3 

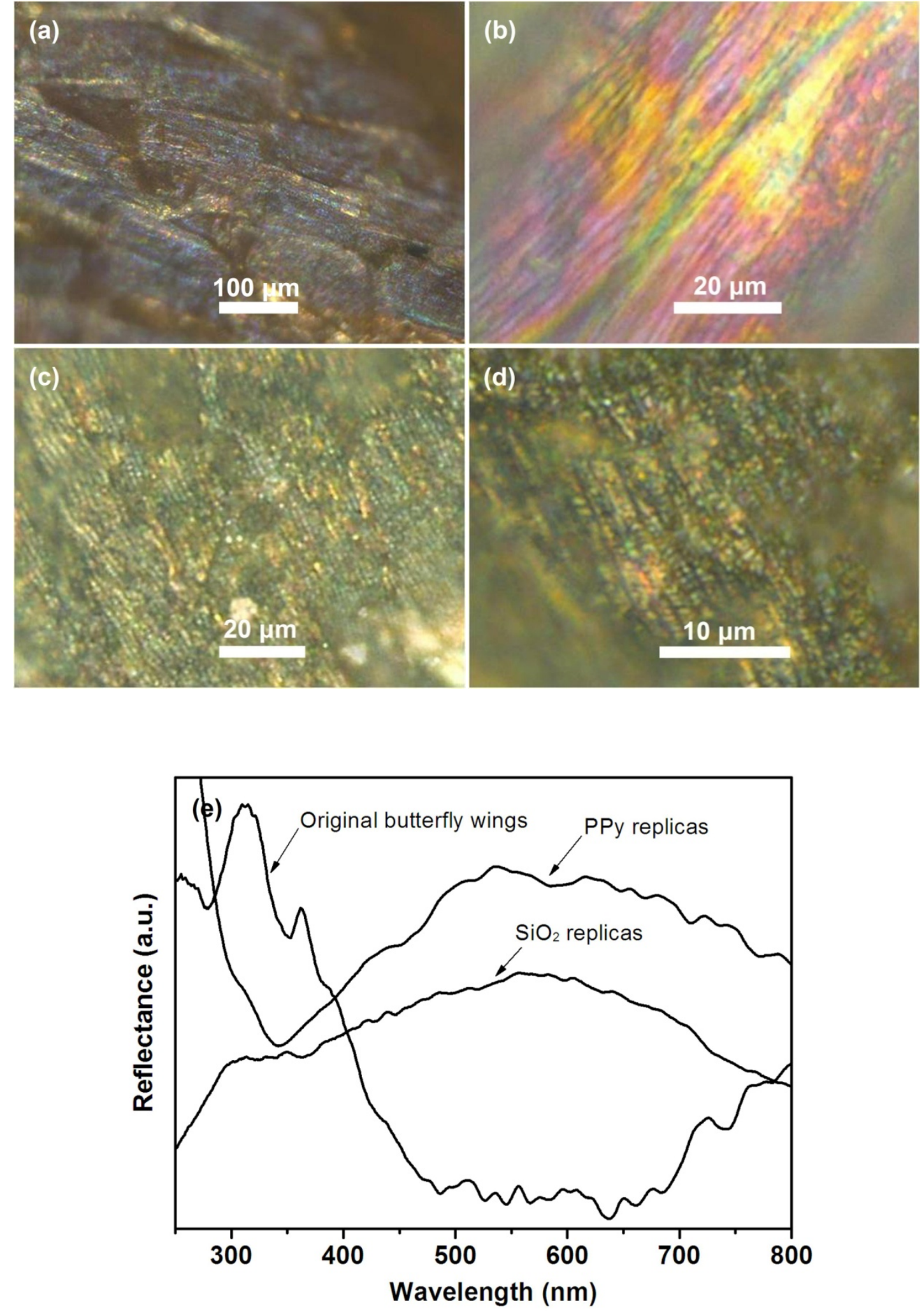

Figure 4 


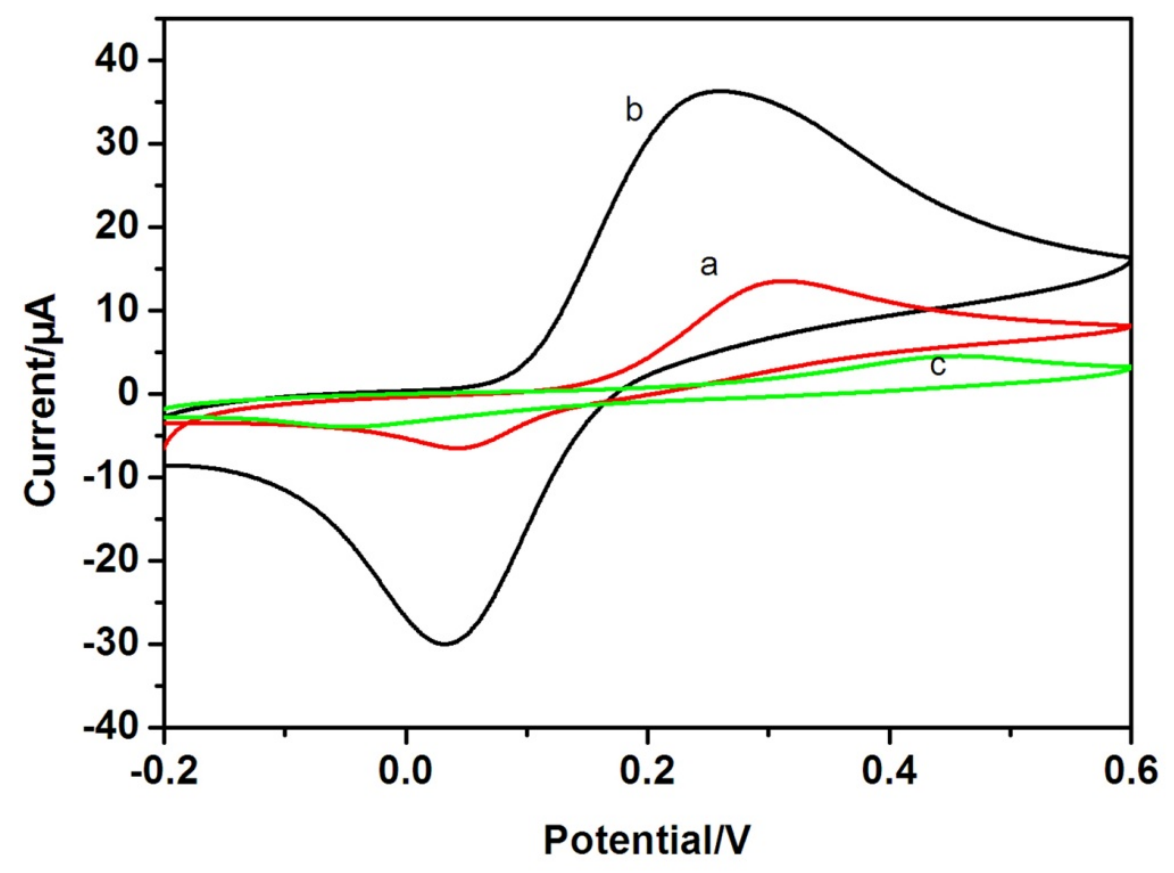

Figure 5 


\section{Supporting Information}

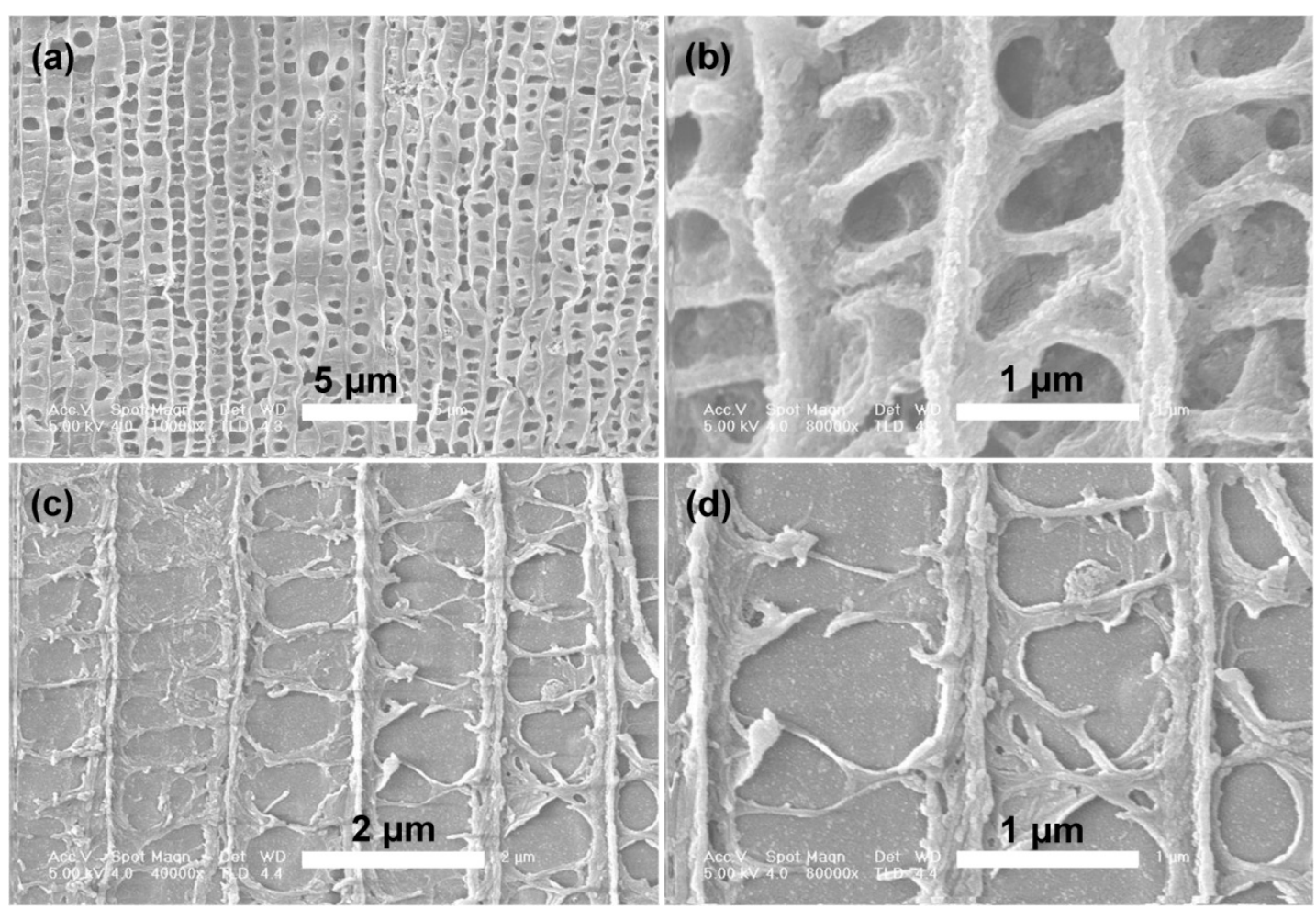

Fig. S1 (a) and (b) FE-SEM images of PNIPA replica before the removal of $\mathrm{SiO}_{2}$ template, (c) and

(d) FE-SEM images of PNIPA replica after the removal of $\mathrm{SiO}_{2}$ template. 